http://revistas.ucr.ac.cr/index.php/ingenieria www.ucr.ac.cr / ISSN 1409-2441
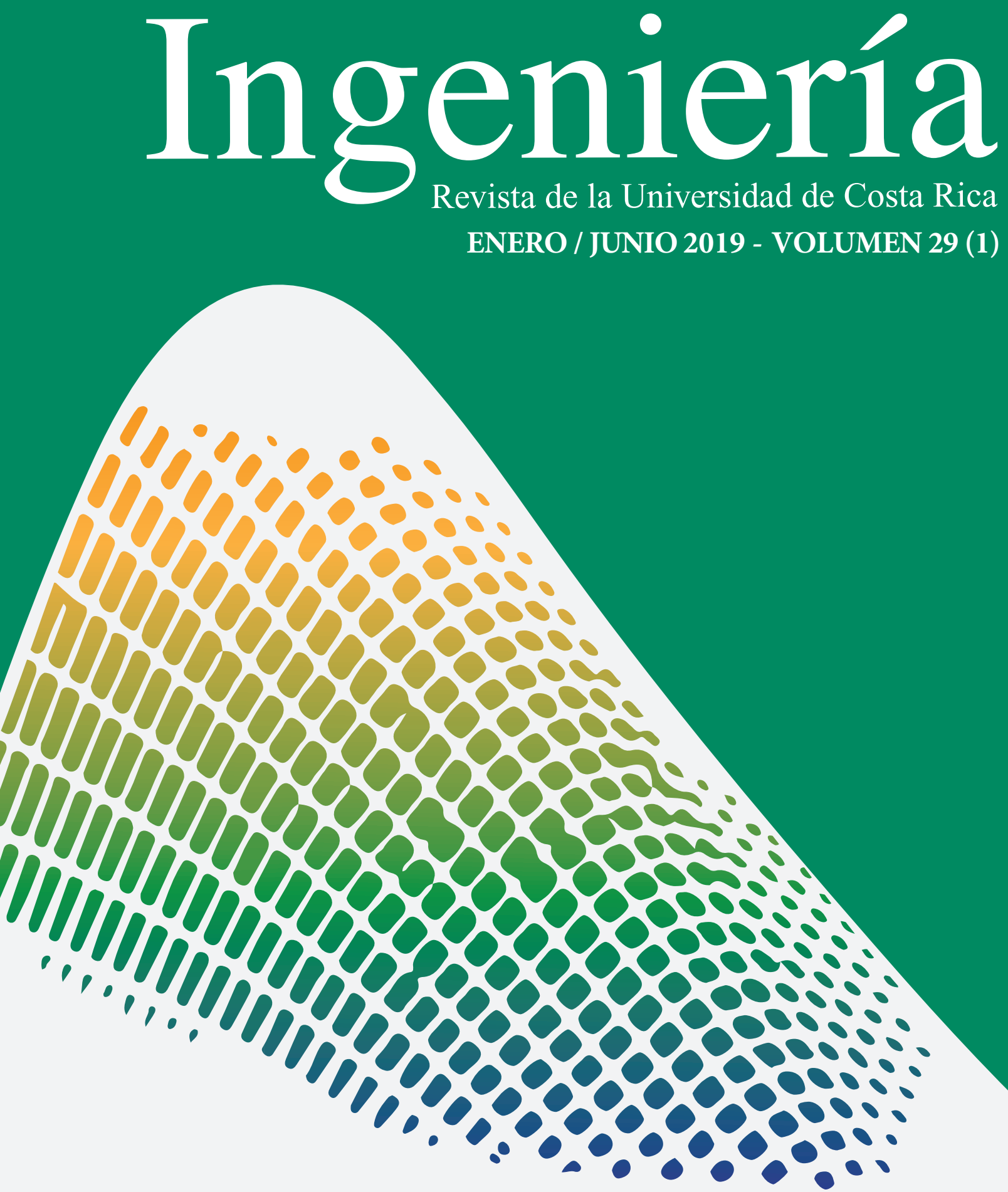


\title{
Extracción de fenol de aguas de residuo utilizando aceite de canola como disolvente orgánico en un proceso de membrana líquida
}

\author{
Phenol extraction from wastewater using canola oil as an \\ organic solvent in a liquid membrane process
}

Eddy Gabriel Jirón García, Yosellyn Guzmán Ramírez, Verónica Hernández Solís, Mary Cruz Bermúdez Rodríguez.

Escuela de Ingeniería Química, Universidad de Costa Rica, San José, Costa Rica

César Augusto Bernal Samaniego, Universidad de Costa Rica, Costa Rica. Sede del Caribe. cesarbernal10@gmail.com

Karina María Rodríguez Mora, Universidad de Costa Rica, San José, Costa Rica. Instituto de Investigaciones en Ingeniería

karina.rodriguezmora@ucr.ac.cr

Recibido: 1 de agosto 2018 Aceptado: 19 de noviembre 2018

\section{Resumen}

Se estudió la extracción de fenol de una disolución simulada utilizando una emulsión de membrana líquida (EML) y un diseño factorial para encontrar las mejores condiciones de operación. Se usó span 80 como surfactante, $\mathrm{NaOH}$ como agente atrapante y tres tipos de fase oleosa: aceite de canola, mezcla aceite de canola/octano y mezcla aceite de canola/parafina. El estudio de las emulsiones simples dio como resultado que las emulsiones más estables fueran: aceite de canola y la mezcla aceite de canola/parafina. Estas son estables por 2 días, mientras que las emulsiones de aceite de canola/octano no son estables, estas se separan. La extracción máxima fue de 77,8 mg/L utilizando la mezcla aceite de canola/parafina y 75,9 mg/L utilizando únicamente aceite de canola como fase oleosa, logrando porcentajes de extracción de $61,1 \%$ y $59,6 \%$ respectivamente. Como resultado del diseño factorial ninguno de los efectos e interacciones fue significativo, lo cual se atribuye a la saturación del agente atrapante.

Palabras clave:

Emulsión, extracción, membrana, fase, transporte. 


\begin{abstract}
In order to find the best operating conditions, the extraction of phenol was studied from a simulate solution by using a liquid membrane emulsion (EML) and a factorial design. Span 80 was used as a surfactant, $\mathrm{NaOH}$ as an encapsulating agent, and three types of oil phase: canola oil, canola oil/octane mixture, and canola oil/paraffin mixture. The study of the simple emulsions gave as result that the more stable emulsions were canola oil and the mixture of canola oil/ paraffin. These are stable for 2 days, while emulsions of canola oil/octane are not, for they get separated. The maximum extraction was the $77,8 \mathrm{mg} / \mathrm{L}$, using the mixture of the oil canola/ paraffin and $75,9 \mathrm{mg} / \mathrm{L}$ by just using canola oil as oil phase, achieving extraction percentages of $61,1 \%$ y $59,6 \%$, respectively. As a result of the factorial design, none of the effects and interactions were significant, which is attributed to the saturation of the encapsulating agent.
\end{abstract}

Keywords:

Emulsions, extraction, membrane, phase, transport. 


\section{INTRODUCCIÓN}

Actualmente, el desarrollo industrial conlleva a un incremento en la producción de residuos, lo cual, a su vez, lleva a una creciente preocupación por la contaminación que se genera. Por lo tanto, lleva a la búsqueda de una implementación de nuevos métodos de dispersión para residuos amigables con el ambiente. Los compuestos aromáticos como el fenol y el clorofenol forman parte de los residuos más comunes, pero son también de los más tóxicos y cancerígenos. Estos, así como una amplia gama de compuestos, están presentes en los efluentes de toda clase de industrias, entre las que se pueden mencionar las refinerías petroleras, las industrias textiles, industrias de plástico, madera e incluso acero (Thayumanavan \& Ellappan, 2014). La concentración de fenoles para las refinerías es $6 \mathrm{mg} / \mathrm{L}-500 \mathrm{mg} / \mathrm{L}$, para procesado de carbón es 2,8 mg/L-1220 mg/L, para productos farmacéuticos, plásticos, pinturas, productos de pulpa, madera y papel es $0,1 \mathrm{mg} / \mathrm{L}-1600 \mathrm{mg} / \mathrm{L}$ (Mohammadi et al., 2014). Por dichas razones encontrar nuevas maneras económicas y eficientes de removerlos es crucial para poder estar en un mercado competitivo y sostenible en términos ambientales.

Para lograr la remoción de los fenoles de las aguas residuales se pueden emplear varios métodos convencionales que, en mayor o menor medida, son eficientes. Algunos de estas tecnologías (como la adsorción y la filtración) se limitan a concentrar los contaminantes transfiriéndolos a otras fases (Mohammad, Orooba , \& Areej , 2017 ). No obstante, pueden resultar costosos y tan eficientes como otros métodos, por ejemplo, las emulsiones de membrana líquida (EML).

De manera aplicada, las EML consisten en un sistema agua/aceite/agua o W/O/W (por sus siglas en inglés) donde el aceite o fase oleosa funciona como una membrana muy selectiva a un compuesto que es encapsulado en la fase acuosa interna (agente atrapante) (Mortaheb, Amini, Sadeghian, Mokhtarani, \& Daneshvar, 2008). Las EML presentan un potencial increíble para operaciones de separación, además de ser una técnica simple de alta eficiencia, de extracción en una sola etapa con área superficial mayor, alcance a funcionamiento continuo y es independiente de la condición de equilibrio (Balasubramanian \& Venkatesan , 2012). Aunado a esto las membranas líquidas no se envenenan y el producto extraído puede ser recuperado al utilizar EML, teniendo así una ventaja económica para aquellas industrias que requieren de fenoles, por ejemplo, en la aplicación como precursores a plásticos, en la producción de adhesivos, colorantes, germicidas y productos intermedios químicos (Parka, Skelland, Forneyb , \& Jae-Hong , 2006). Sin embargo, la desventaja de este método es que las emulsiones no son termodinámicamente estables. Por esta razón es importante el estudio de los parámetros que mejoran la estabilidad de una emulsión y, por ende, la eficiencia y la extracción. Variables como la fase oleosa, concentración de surfactante, velocidad y tiempo de emulsificación, así como el tiempo de extracción son algunas de las variables que se pueden modificar para beneficiar las operaciones de extracción (Bahloul, Bendebane, Djenouhat, Meradi, \& Ismail , 2016).

En este artículo se propone sustituir la fase oleosa de la emulsión, la cual comúnmente es un derivado del petróleo como n-heptano, queroseno o dicloroetano, por aceite de canola y una mezcla de aceite de canola y parafina. Esto dado a que el aceite 
de canola es fácil de conseguir y es menos viscoso que otros aceites (como el de soya, maíz o ricino que son los que se consiguen comercialmente), haciendo que el aceite de canola tenga mayor potencial para la emulsificación y para la extracción. Así, no solo se podría extraer fenoles de aguas residuales con la posibilidad de recuperarlos, sino también que se estaría implementando un método eficiente, barato, innovador y verde.

\section{MATERIALES Y MÉTODOS}

\subsection{Materiales}

Los materiales empleados son; agua destilada, fenol, hidróxido de sodio, parafina, octano, span 80, ácido sulfúrico, ácido nítrico, amoniaco y aceite de canola.

\subsection{Metodología}

La metodología utilizada se expondrá a continuación.

\subsubsection{Formulación de emulsiones simples:}

Se realizó un barrido de formulación de emulsiones utilizando span 80 al 2\% como surfactante y variando la fracción de aceite, como se muestra en la tabla 1. En el caso de fase oleosa se emplean tres diferentes fases: aceite de canola $(\mathrm{C})$, aceite de canola con octano en relación 1:1 (OC) y aceite de canola con aceite de parafina relación 1:1(CP). Esto con la finalidad de lograr diferentes membranas. En el caso de la fase acuosa se empleó $\mathrm{NaOH} 0,1 \mathrm{~mol} / \mathrm{L}$.

El método de mezclado utilizado fue de baja energía, se usó un agitador magnético a una velocidad de $1400 \mathrm{rpm}$ durante $3 \mathrm{~min}$. El procedimiento fue mezclar en primera instancia el surfactante con el aceite e iniciar agitación; luego, se agregó lentamente la fase acuosa, continuando la agitación hasta cumplir los $3 \mathrm{~min}$.

Tabla 1. Composición de las emulsiones.

Relaciones de agua y aceite para cada barrido de formulación.

\begin{tabular}{ccc}
\hline Identificación & $\begin{array}{c}\text { Fracción de } \\
\text { Aceite }\end{array}$ & Fracción de agua \\
\hline 1 & 80 & 20 \\
2 & 75 & 25 \\
3 & 70 & 30 \\
4 & 65 & 35 \\
5 & 55 & 45 \\
\hline
\end{tabular}




\subsubsection{Prueba de estabilidad de las emulsiones simples:}

Para la determinación de la estabilidad se realizó una observación cualitativa a las $2 \mathrm{~h}$ y las $48 \mathrm{~h}$, con la finalidad de apreciar alguna separación de la emulsión y poder evaluar si existía sedimentación y flotación creaming). Se realizó la toma de fotos al microscopio para poder tener un parámetro de partida del tamaño de gota y poder ver explicar los fenómenos observados macroscópicamente, para ello se empleó un microscopio eclipse $80 \mathrm{i}$ del NIS elements ${ }^{1}$.

\subsubsection{Tamaño de partícula emulsión simple:}

Para realizarla determinación del tamaño de partícula se procedió a hacer una dilución de la emulsión en hexano (esto debido a que las fases externas de parafina o canola imposibilitaban la medición por láser). Seguidamente, se empleó el Mastersizer 2000, serie MAL 1051095. Estas mediciones se realizaron para las emulsiones a las cuales se les hizo el proceso de extracción.

\subsubsection{Estabilidad emulsión simple:}

Se determinó la transmisión y la reflexión de la luz con respecto al tiempo, generando gráficas que indican cuál de los fenómenos ocurre en las emulsiones estudiadas, ya sea clarificación, sedimentación o coalescencia. Para esto se empleó el turbiscan MA2000² (Mengual, Meunier, Cayré, Puech, \& Snabre, 1999).

\subsubsection{Determinación de nitrofenol:}

Se realizó una curva de calibración en un espectrofotómetro utilizando patrones de nitrofenol a concentraciones de 9,9 mg/L; 24,8 mg/L; 49,7 mg/L; 74.5 mg/L; 99,3 $\mathrm{mg} / \mathrm{L} ; 124,2 \mathrm{mg} / \mathrm{L} ; 149,0 \mathrm{mg} / \mathrm{L}$, midiendo la absorbancia a $400 \mathrm{~nm}$ (Stoughton, 1936).

\subsubsection{Extracción:}

Se separó la emulsión múltiple por medio de centrifugación. Lo anterior se realizó en un equipo marca Clay Adams Sero-fuge durante un tiempo de 10 min a 3000rpm. Posteriormente, se tomó la fase acuosa de la emulsión simple y se procedió a determinar la concentración de nitrofenol por absorbancia UV.

\subsubsection{Diseño experimental:}

Se realizó una emulsión múltiple al verter la emulsión simple en una disolución de fenoles con una concentración de 127,32 mg/L. Se optó por utilizar una factorial 23 utilizando el nivel de agitación a $1800 \mathrm{rpm}$ y a $1400 \mathrm{rpm}$, el tiempo de extracción a 5 minutos y 7 minutos, y la fase oleosa como factores. Además, se utilizó la diferencia de concentración como posible respuesta. La finalidad de emplear diferentes velocidades en la agitación y tiempo de contacto era ver si alguna de ellas favorecía la transferencia de masa a la fase acusa interna. En el caso de la variable de composición de la fase oleosa se debe a diferencias en viscosidad y por lo tanto cambio de membrana. 

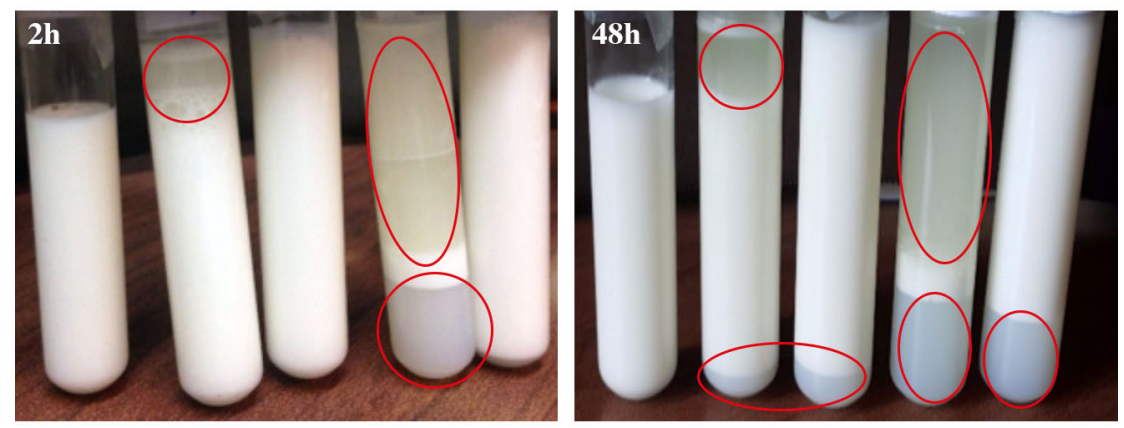

Figura 1. Determinación cualitativa de estabilidad para el barrido de aceite de canola (C1, C2, C3, C4 y C5 de izquierda a derecha).

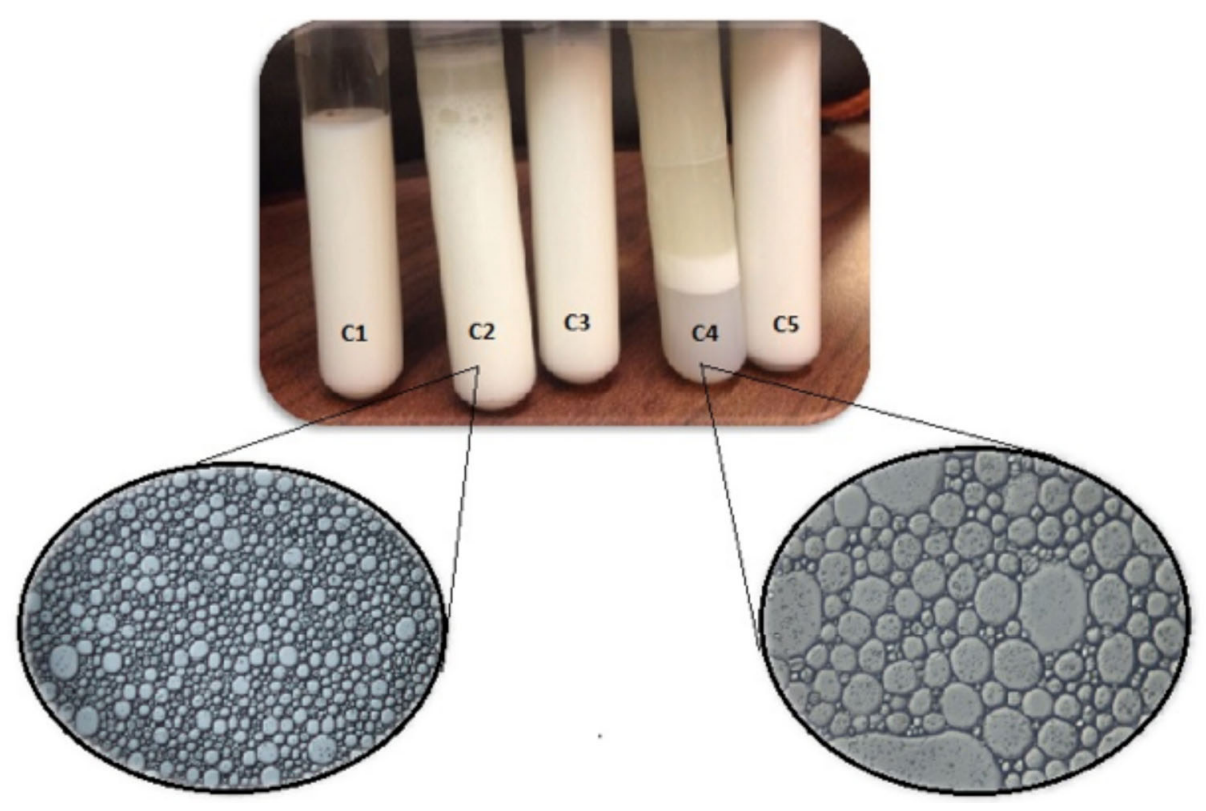

Figura 2. Visión microscópica de las emulsiones de aceite de canola.
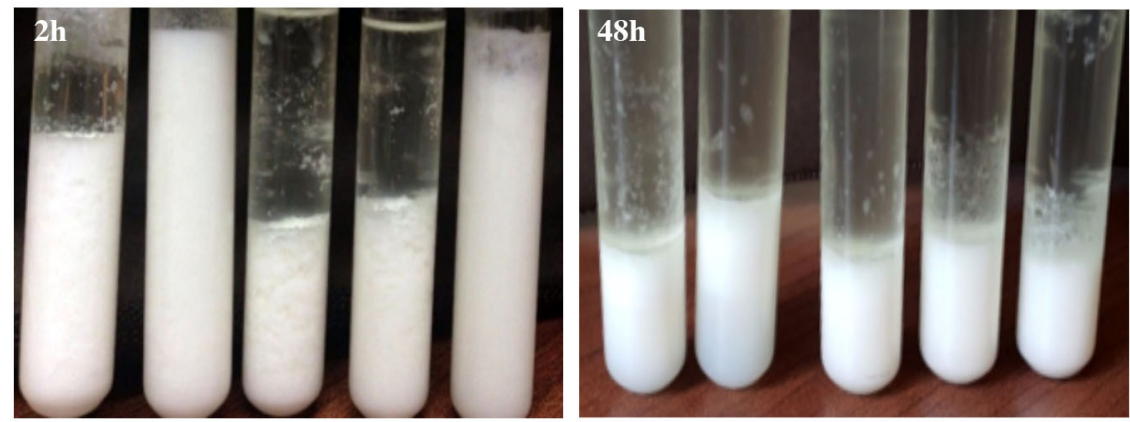

Figura 3. Determinación cualitativa de estabilidad para el barrido de aceite de canola/octano (OC1, OC2, OC3, OC4 y OC5 de izquierda a derecha). 


\section{RESULTADOS Y DISCUSIÓN}

\subsection{Emulsión simple}

Se procedió a realizar el análisis de las emulsiones simples. El surfactante elegido es de HLB bajo, dado que lo que se requiere es la estabilización de la fase oleosa como fase externa (Gennaro, 2006). Se decide realizar las pruebas de estabilidad tanto cualitativas como cuantitativas usando $2 \mathrm{~h}$ como tiempo mínimo, debido a la aplicación porque el proceso de extracción toma un tiempo determinado para poder realizarse, y $48 \mathrm{~h}$ como tiempo máximo, pensando en que si hubiese separación la emulsión podría redispersarse y aplicarse. En la Figura 1 se puede notar cómo en las emulsiones, empleando solo canola como fase oleosa, $\mathrm{C} 1$ y C3 y C5 permanecen estables a las $2 \mathrm{~h}$. Mientras que, a las $48 \mathrm{~h}$ se denota que solo permanece estable la emulsión C1. A las $2 \mathrm{~h}$ y las $48 \mathrm{~h}$ se ven procesos de clarificación. Asimismo, a las $2 \mathrm{~h}$ se ve en C2 sedimentación y en C4 flotación o creaming. A las 48h se nota en C3 y C5 un proceso de clarificación solamente en la parte inferior, mientras que en $\mathrm{C} 2$ y $\mathrm{C} 4$ se da el proceso de clarificación en la parte superior e inferior. Marco en rojo se ve en la siguiente figura las fracciones que se han separado de la emulsión.

En la Figura 2, se muestra una imagen de las diferencias en el tamaño de gota de las emulsiones recién elaboradas. Como se puede observar, la $\mathrm{C} 4$ posee un tamaño de gota más grande, pues ambas están tomadas con la misma magnificación. Al poseer mayor tamaño de gota se da el favorecimiento de la coalescencia, de tal forma que las gotas se juntan para formas gotas de mayor tamaño, como la que se muestra en la parte interior izquierda, esto ocasiona que la emulsión pierda la estabilidad y se separe.

Otras de las posibles membranas que se estudiaron fue la mezcla de aceite de canola/ octano. Se realizó la emulsificación y se estudió el tiempo de separación. En este caso, la separación para todas las emulsiones fue inmediata. De acuerdo con la Figura 3; esto se puede atribuir a la baja viscosidad del octano en comparación con el aceite de canola, por lo que el surfactante no puede estabilizar la emulsión y esta se rompe. En este caso las más inestables son las OC1, OC3 y OC4.

Si se observa la Figura 4 y se compara el tamaño de gota de la emulsión más estable OC5 y de las menos estables OC 3 , se denota que la OC3 es tan inestable que coalesce al medirla y por esa razón se ven solo gotas parciales, y se puede notar que lo que se observa en la imagen es la fracción más pequeña. En el caso de OC5 se observar homogénea y con gotas medianas, por tanto, es la más estable de este barrido de formulación.

La siguiente modificación de la fase oleosa se realizó con aceite de canola y parafina. En este caso, las emulsiones son muy estables, de acuerdo con la Figura 5, las emulsiones no se rompen con facilidad y son mucho más estables que las emulsiones que poseen solo aceite de canola en su composición. Solamente se ve una ligera separación en la CP3, la cual se encuentra señalada con rojo.

$\mathrm{Al}$ ver el tamaño de gota de una de ellas se observa que es bastante pequeño y eso favorece la estabilidad de la emulsión. 


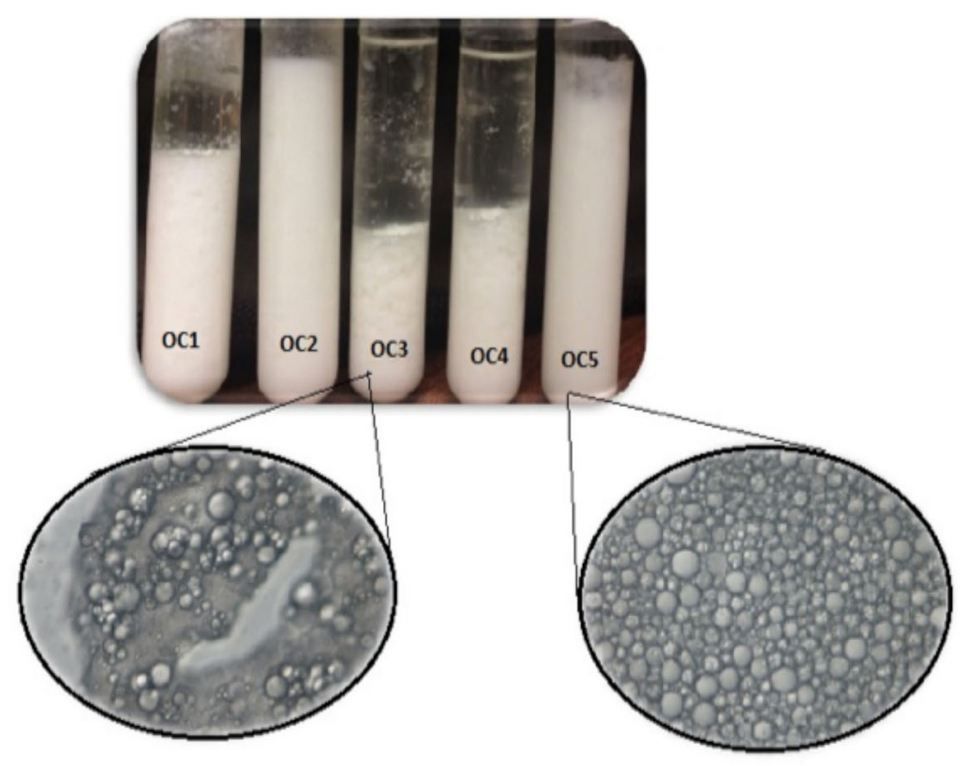

Figura 4. Determinación de la estabilidad de las emulsiones utilizando aceite de canola y octano como fase oleosa.
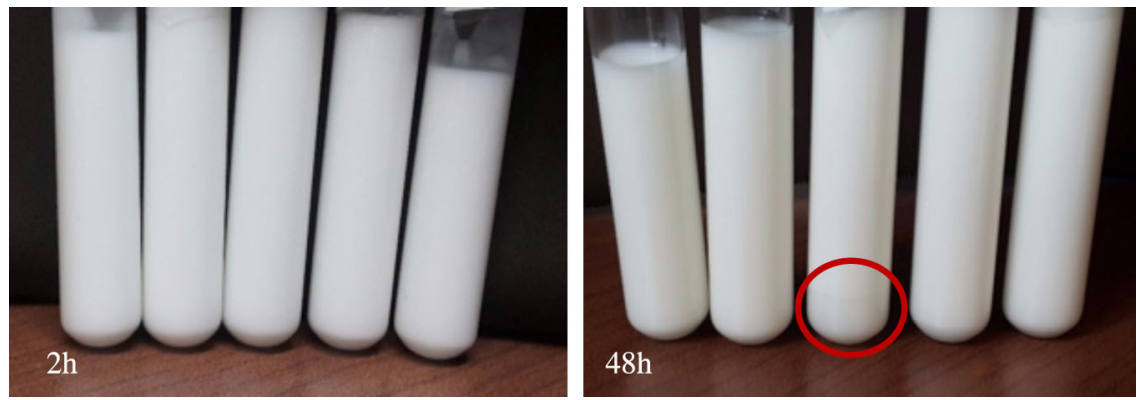

Figura 5. Determinación cualitativa de estabilidad para el barrido de aceite de canola/parafina (CP1, $\mathrm{CP} 2, \mathrm{CP} 3, \mathrm{CP} 4$ y $\mathrm{CP} 5$ de izquierda a derecha).

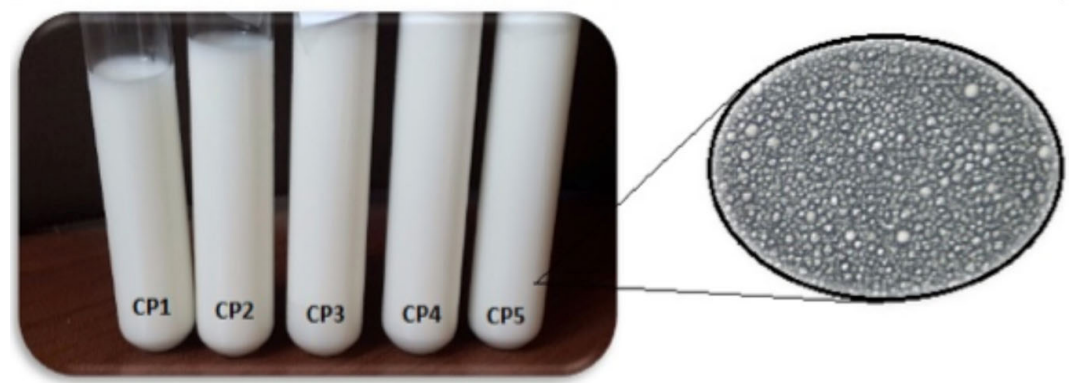

Figura 6. Determinación de la estabilidad de las emulsiones utilizando aceite de canola y parafina como fase oleosa. 
Seguidamente, se eligieron las emulsiones más estables para realizar el proceso de extracción, C1 y CP1. No obstante, la CP1 queda posteriormente descarta debido a que no puede realizar la extracción, pues se quiere que esta forma una emulsión múltiple para que haya difusión del compuesto de interés de la fase acuosa externa (medio contaminado) a la fase acuosa interna y así poder encapsularlo, pero al hacer la prueba con la CP1 se da una inversión de la fase y queda una emulsión simple de aceite en agua, perdiendo por completo el sentido de encapsulación. Por esta razón se decide usar la CP5, emulsión que seguía en orden por estabilidad.

Posteriormente, se procede a caracterizar las emulsiones de trabajo C1 y CP5. Para ellas se realiza el tamaño de partícula y vemos que para $\mathrm{C} 1$ está en $11 \mu \mathrm{m}$, mientras que la CP5 presenta dos modas una cerca de $0,2 \mu \mathrm{m}$ y $20 \mu \mathrm{m}$. En el caso del CP5, esta presenta problemas para ser medido, pues no logra dispersarse tan bien en hexano como la $\mathrm{C} 1$. No obstante, se cree que este valor está un poco alejado del valor real y la curva debería estar más baja que la $\mathrm{C} 1$, pero por los problemas de disolución se generan gotas más grandes.

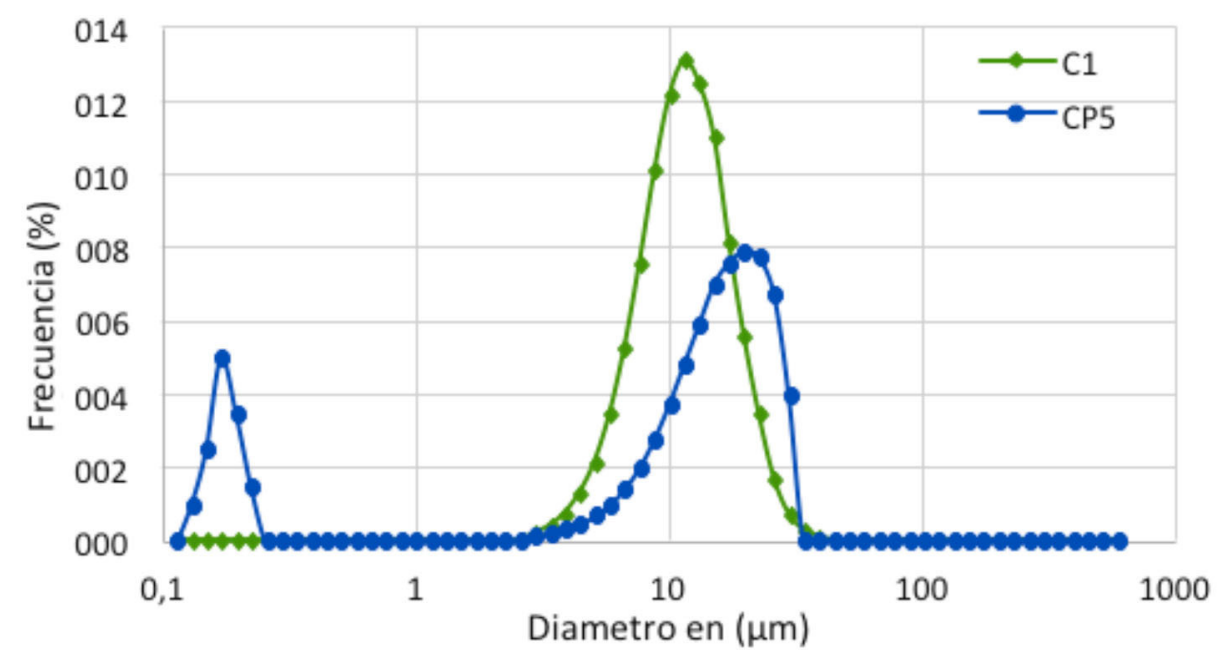

Figura 7. Determinación de la estabilidad de las emulsiones utilizando aceite de canola y parafina como fase oleosa

Se comparó la estabilidad de las emulsiones OC1, C1 y CP5. En el caso de las C1 y las CP5 se nota una leve clarificación al final de la gráfica (ver Figura S6). En el caso de OC1 se muestra un comportamiento diferente. Esta se ve totalmente inestable y se ve sedimentación y clarificación. Ante esto, se propuso graficar el comportamiento de cada una para observar la estabilidad dependiendo de las dimensiones del tubo de medida y el comportamiento de la Figura S6.

En la figura 8 es posible observar cómo la emulsión OC1, no presenta estabilidad en ninguna región del tubo. Pues como se muestra en su gráfica posee una transmitancia fluctuante. En el caso de C1 y CP5 se muestra que la mayoría del tubo de 0-36mm 
se encuentra totalmente estable, mientras que de $36-40 \mathrm{~mm}$ se muestra cambios considerables. Sin embargo, en el caso de CP5 a los 20 min se da una estabilización, pero a mayores valores, esto puede deberse a la formación de agregados en dicha zona que causan el efecto de la clarificación vista en la figura S6. Mientras, que para los cambios en la $\mathrm{C} 1$ de igual forma se puede asociar a un aumento en el tamaño de partícula que genera el efecto de clarificación visto en la figura S6, con la diferencia de que no se observa que se estabilice en el tiempo. Es importante destacar que en ambos casos este fenómeno es imperceptible a la vista.

\subsection{Extracción}

Para realizar la cuantificación del fenol se cabo una curva de calibración utilizando nitrofenol de acuerdo con la ecuación 1 y se procedió a realizar la medición a 400 nm.

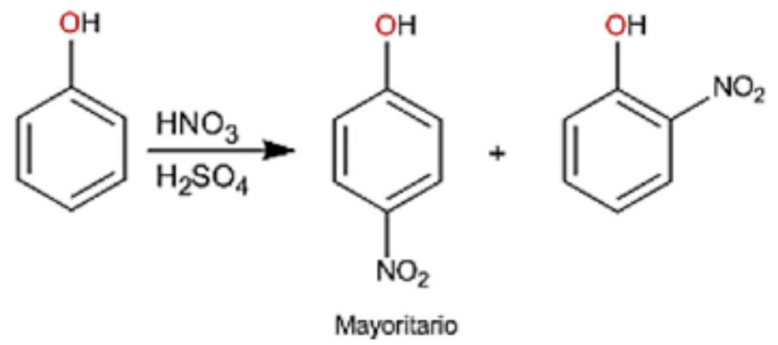

Una vez hecha la coloración se procedió a realizar una curva de calibración (Figura 9). Para obtener, por medio de la Ley de Beer, la relación entre absorbancia y concentración. Esta curva funciona en este caso en especial ya que la reacción de la cual depende la extracción es con el grupo $\mathrm{OH}-$ del fenol.

Posteriormente, se realizó el proceso de extracción formando una emulsión múltiple W/O/W dispersando la emulsión simple en una matriz (en este caso nitrofenol) con agitación constante (Cardenas \& Salager, 2011).De esta forma, la emulsión simple se dispersó y formó una membrana permeable para el fenol.

Se utilizó la CP5 y la C1 para el proceso de extracción. Esto porque las emulsiones múltiples más estables se obtuvieron con una emulsión simple de aceite de canola con composición C1 y una membrana de composición CP5 agitando a $200 \mathrm{rpm}$ por 5 minutos ambas con una proporción de 1:2.5 emulsión simple: nitrofenol.

Como la EML posee dos componentes en su fase oleosa, actúan dos fenómenos de transporte. El primero de ellos es la difusión causado por la diferencia de concentración a ambos lados de la membrana. De acuerdo con la ecuación (2), el fenol difunde a través de la membrana y llega a reaccionar con el $\mathrm{NaOH}$ para formar fenolato de sodio y queda atrapado dentro de la membrana (Othman, y otros, 2017).

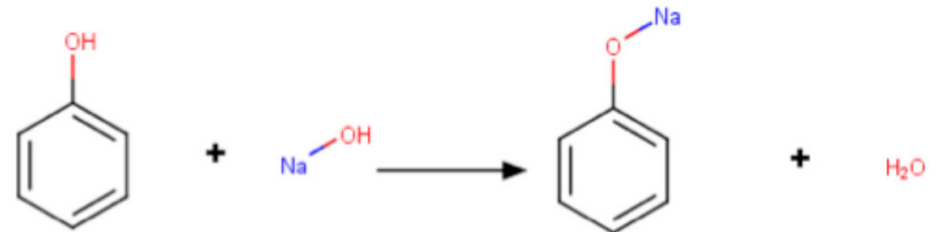




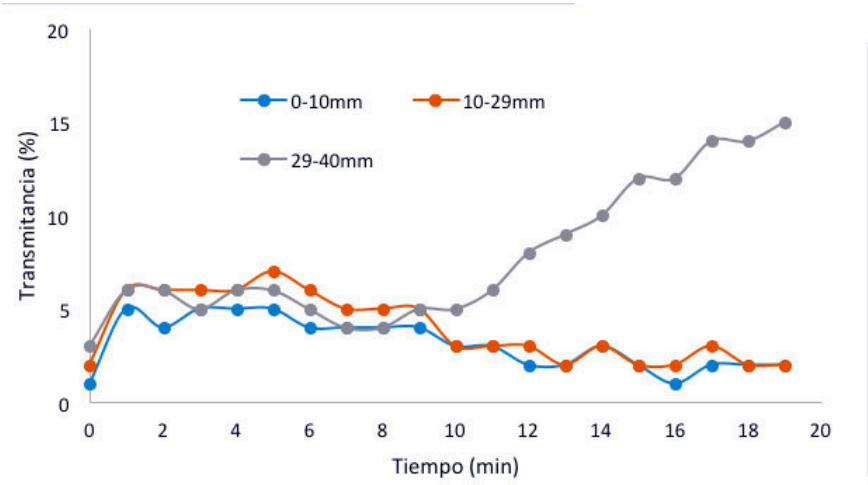

A) Emulsión OC1.

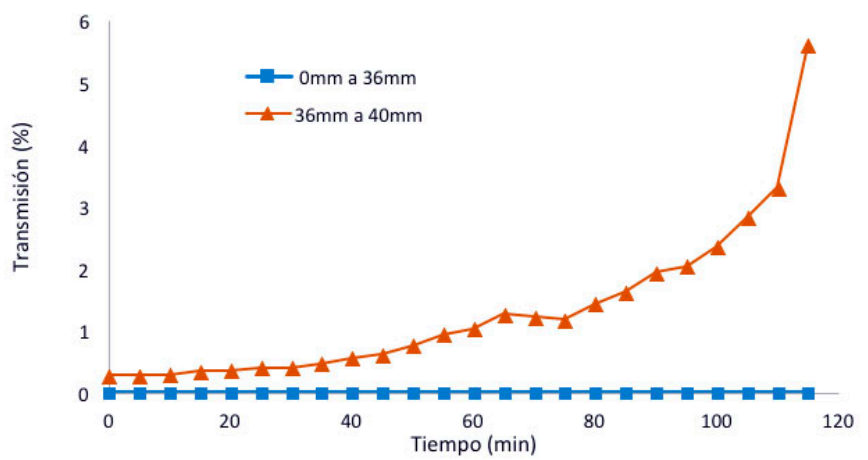

B) Emulsión C1.

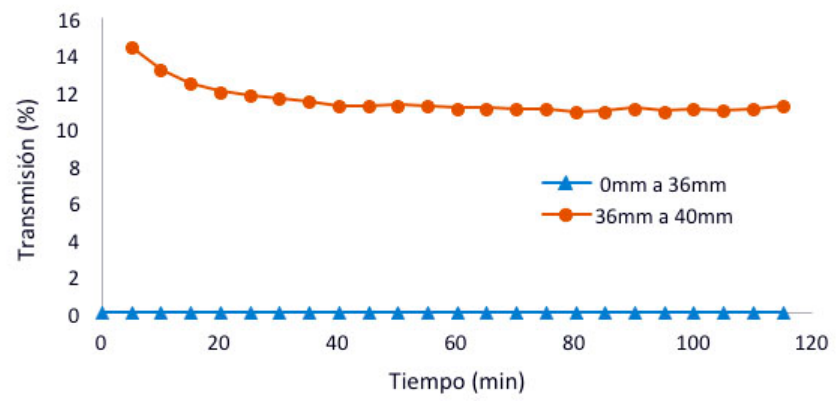

C) Emulsión CP5.

Figura 8. Determinación de la estabilidad de las emulsiones A) a los 20 min B) y C) 2 horas. 
El mecanismo presente cuando se utilizan aceite de canola es denominado transporte, el mecanismo se presenta en la figura 10. En este fenómeno, el fenol reacciona en un lado de la interface con los triglicéridos del aceite (TG) formando una complejo fenol-triglicérido (Ph-TG), el cual se desplaza hacia el otro lado de la membrana y es liberado. Luego, al reaccionar con el $\mathrm{NaOH}$ y romperse los puentes de hidrógeno que formaban el complejo y formar fenolato de sodio, el cual no puede pasar nuevamente a través de la membrana. De esta manera el triglicérido queda libre para repetir (Othman, y otros, 2017).

\subsection{Análisis}

Se realizó un diseño factorial con tres réplicas, ya que se requieren al menos dos réplicas para analizar adecuadamente los efectos e interacciones presentes (Garza Villegas, 2013). Utilizando dos niveles y tres factores, los cuales son presentados en la Tabla 2. Los niveles bajos fueron establecidos de las condiciones de preparación de las emulsiones múltiples. A partir de ello, se consideró que al aumentar el nivel de agitación se incrementa el área superfical de la extracción. Mientras que al aumentar el tiempo de extracción es posible mejorar la extracción hasta llegar a un equilibrio. Finalmente, al cambiar el tipo de membrana se puede corroborar si al existir dos fenómenos involucrados la extracción se hace mas eficiente. Se debe recalcar que el objetivo del factorial es encontrar las condiciones que maximicen la extracción.

Tabla 2. Niveles y factores utilizados en el diseño factorial.

\begin{tabular}{llcc}
\hline & & \multicolumn{2}{c}{ Niveles } \\
& & Alto & Bajo \\
\hline A & Nivel de agitación / (rpm) & 1800 & 1400 \\
B & Tiempo de extracción / (min) & 7 & 5 \\
C & Tipo de membrana & Aceite de canola & Aceite de canola/parafina \\
\hline
\end{tabular}

El cambio de concentración determinado correspondió a la extracción realizada. La extracción máxima fue de $77,778 \mathrm{mg} / \mathrm{L}$ utilizando aceite de canola con parafina y $75,925 \mathrm{mg} / \mathrm{L}$ utilizando únicamente aceite de canola como fase oleosa, logrando porcentajes de extracción de $61,07 \%$ y 59,62\% respectivamente. El resultado del análisis factorial con tres réplicas y una significancia de 9,774 es que ninguna de las interacciones o efectos es significativa, lo cual se puede comprobar en la Figura 10. Dado que ninguno de los efectos o interacciones estandarizados es mayor que el valor de la "t-Student” en el gráfico de Pareto (Gutiérrez, 2008).

La no significancia de los efectos e interacciones se puede atribuir a la saturación del agente atrapante. En otras palabras, el tiempo de extracción es tan largo para este sistema que el $\mathrm{NaOH} 0.1 \mathrm{~mol} / \mathrm{L}$ reacciona por completo, por lo que, aunque se aumente 


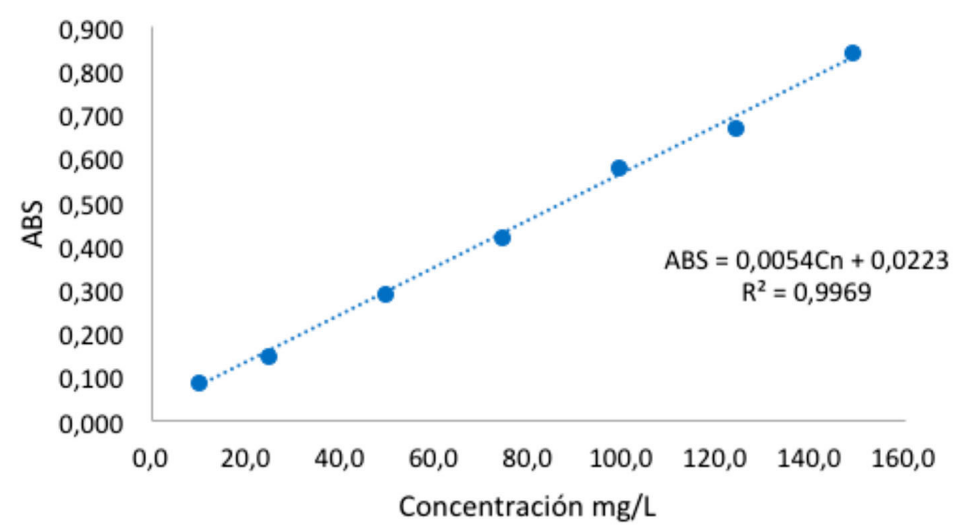

Figura 9. Curva de calibración para obtener la concentración de fenol.

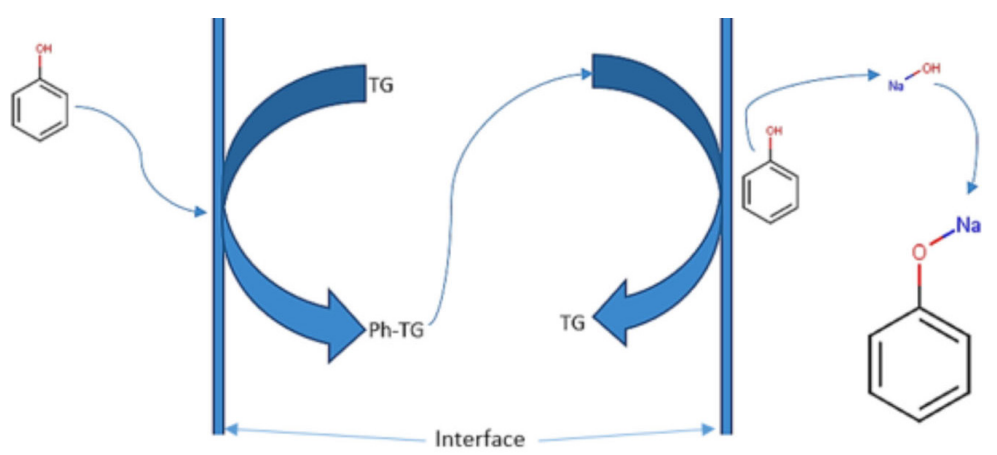

Figura 10. Mecanismo de transporte de fenol a través de la membrana de aceite, modificado a partir de Badgujar \& Rastrogi (2011).

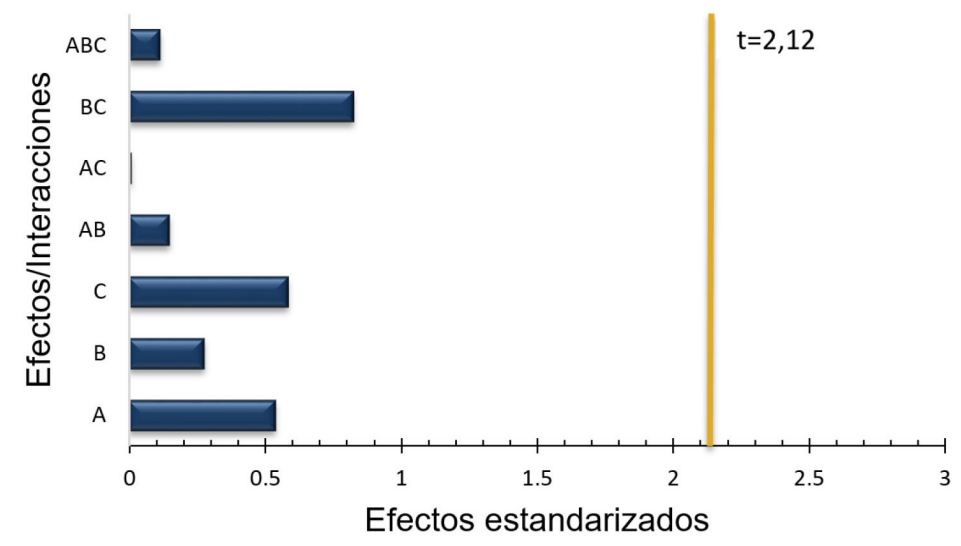

Figura 11. Gráfico de Pareto. 
la velocidad de agitación o el tipo de fase oleosa, no se podría maximizar la extracción. Es importante recalcar que el factorial se realizó en este punto para poder ver los efectos específicamente en el proceso de extracción.

\section{CONCLUSIONES}

- Las emulsiones simples más estables se obtienen con la fase oleosa de aceite de canola C1 y C5, la formulación 2 es descartada ya que el octano y el aceite de canola no es compatible.

- Las emulsiones múltiples más estables se obtuvieron con una emulsión simple de aceite de canola con composición C1 y una membrana de composición CP5 donde se agitó a $1400 \mathrm{rpm}$ por 5 minutos, ambas con una proporción de 1:2.5 emulsión simple: nitrofenol.

- Se obtuvo una curva de calibración para medición de fenol, convirtiéndolo a nitrofenol, en un intervalo de $9,9 \mathrm{~m} / \mathrm{L}$ a $149 \mathrm{mg} / \mathrm{L}$ con un coeficiente de correlación lineal de $0,9969$.

- La extracción máxima fue de $77,78 \mathrm{mg} / \mathrm{L}$, donde se utilizó la mezcla aceite de canola/parafina y 75,93 mg/L, usando únicamente aceite de canola como fase oleosa, logrando, a su vez, porcentajes de extracción de $61,1 \%$ y 59,6\% respectivamente.

- Para una significancia de 9,774 se tiene que los efectos e interacciones no resultan significativos, por lo que las mejores condiciones se obtienen al utilizar solo aceite de canola como disolvente orgánico, una velocidad de extracción de 1400 rpm y un tiempo de extracción de 5 min.

\section{NOTAS}

1 Ver información suplementaria S1, S2 y S3

2 Ver información suplementaria S4 y S5

\section{REFERENCIAS}

Badgujar, V., \& Rastrogi, N. (2011). Extraction of phenol from aqueous effluent usin triglycerides in supported liquid membrane. Desalin Water Treat(36), 187-196.

Bahloul, L., Bendebane, F., Djenouhat, M., Meradi, H., \& Ismail , F. (2016). Effects and optimization of operating parameters of anionic dye extraction from an aqueous solution using an emulsified liquid membrane: Application of designs of experiments. Journal of the Taiwan Institute of Chemical Engineers(59), 26-32.

Balasubramanian , A., \& Venkatesan , S. (2012). Optimization of process parameters using response surface methodology for the removal of phenol by emulsion liquid membrane. Polish Journal of Chemical Technology, 46-49.

Cardenas, A., \& Salager, J. (2011). Cuaderno FIRP S277-C Emulsiones Múltiples. Universidad de los Andes.

Garza Villegas, J. (2013). Experiment design application for analysis of the drying a product. Innovaciones de Negocios(19), 145-158. 
Gennaro, A. (2006). Remigton Farmacia. Buenos Aires: Panamericana.

Gutiérrez, H. (2008). Análisis y diseño de experimentos. México: McGraw-Hill.

Mohammad, F., Orooba , N., \& Areej , F. (2017). Study on removal of phenol from synthetic wastewater using solar photo catalytic reactor. Journal of King Saud University - Engineering Sciences.

Mohammadi, S., Kargari, A., Sanaeepur, H., Abbassinan, K., Najafi, A., \& Mofarrah, E. (2014). Phenol removal from industrial wastewaters: A short review. Desalin Water Treat, 1-20.

Mengual, O., Meunier, G., Cayré, I., Puech, K., \& Snabre, P. (1999). TURBISCAN MA 2000: Multiple light scattering measurement for concentrated emulsion and suspension instability analysis. Talanta, 50(2), 445-456.

Mortaheb, H., Amini, M., Sadeghian, F., Mokhtarani, B., \& Daneshvar, H. (2008). Study on a new surfactant for removal of phnol from wastewater by emulsion liquid membrane. J. Hazard. Mater(160), 582-588.

Othman, N., Mohamed, N., Yin Shu, L., Jusoh, N., Idroas, M., \& Masahiro, G. (2017). Easy removing of phenol from wastewater using vegetable oil-based organic solvent in emulsion liquid membrane process. Chinese Journal of Chemical Enginnering(25), 45-52.

Parka, Y., Skelland, A., Forneyb, L., \& Jae-Hong, K. (2006). Removal of phenol and substituted phenols by newly developed emulsion liquid membrane process. Water Research (40), 1763-1772.

Stoughton, R. (1936). A method for the quantitative determination of phenols. Journal of biological chemistry(1), 293-298.

Thayumanavan, P., \& Ellappan, K. (2014). Removal of phenols from acidic environment by horseradish peroxidase (HRP): Aqueous thermostabilization of HRP by polysaccharide additives. Journal of the Taiwan Institute of Chemical Engineers(45), 625-634. 


\section{ANEXOS}

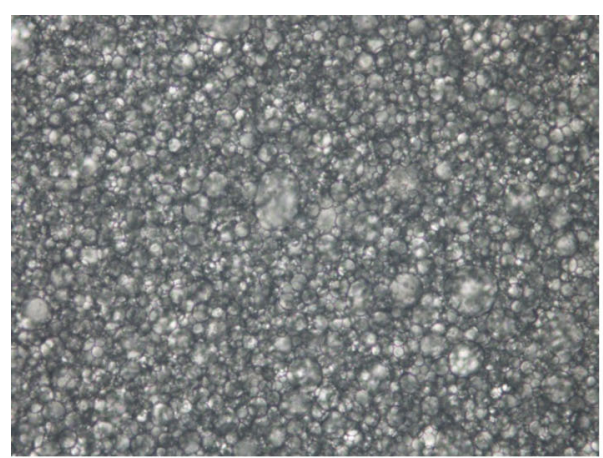

OC1. Magnificación 20X

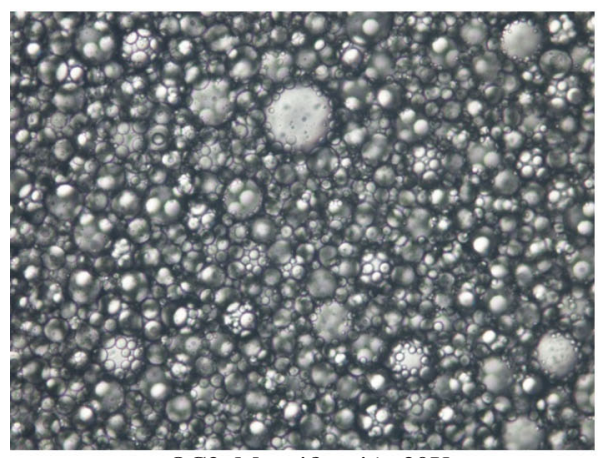

OC3. Magnificación 20X

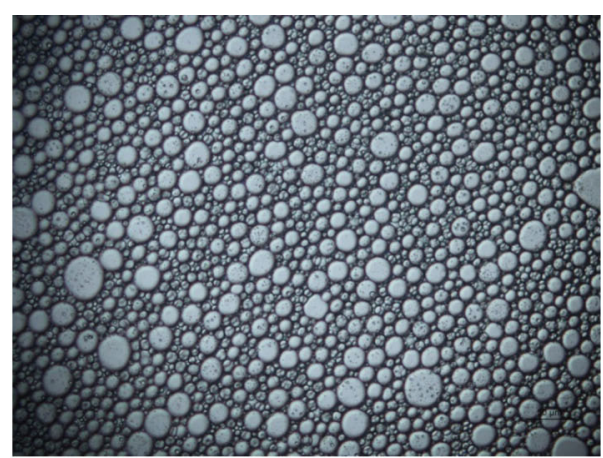

OC2. Magnificación 20X

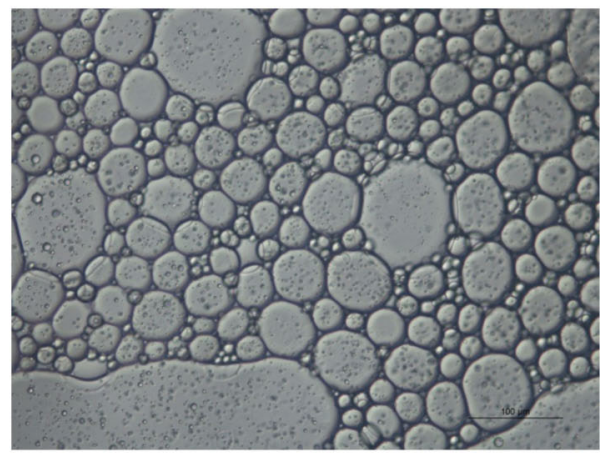

OC4. Magnificación 20X

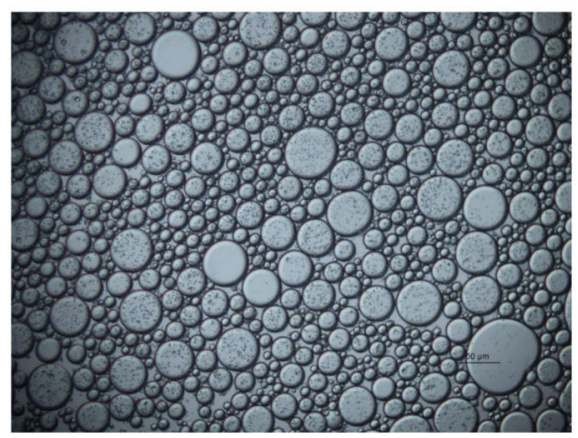

OC5. Magnificación 20X

Figura S2. Fotos del microscopio de las diferentes emulsiones para la fase oleosa de aceite de canola 


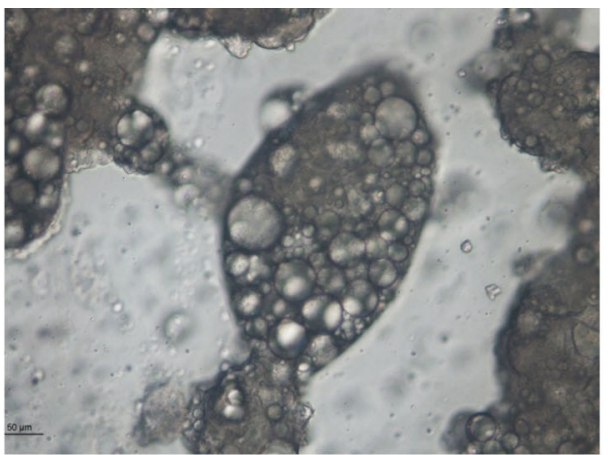

OC1. Magnificación 20X

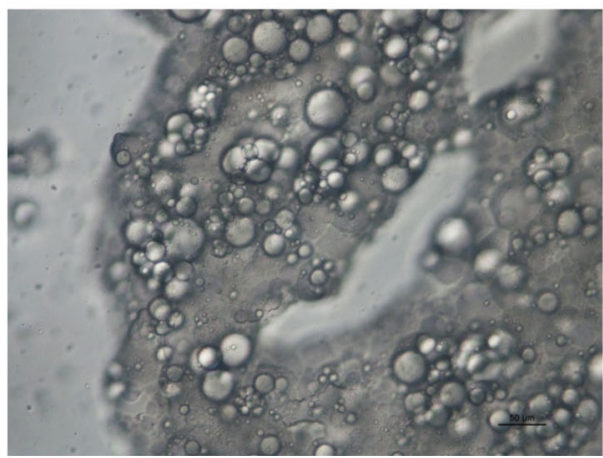

OC3. Magnificación 20X

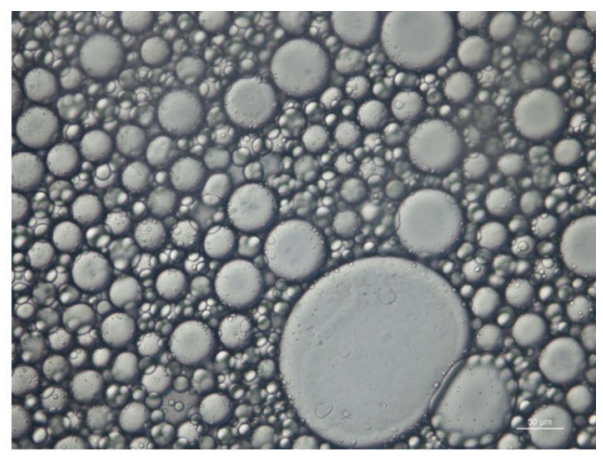

OC2. Magnificación 20X

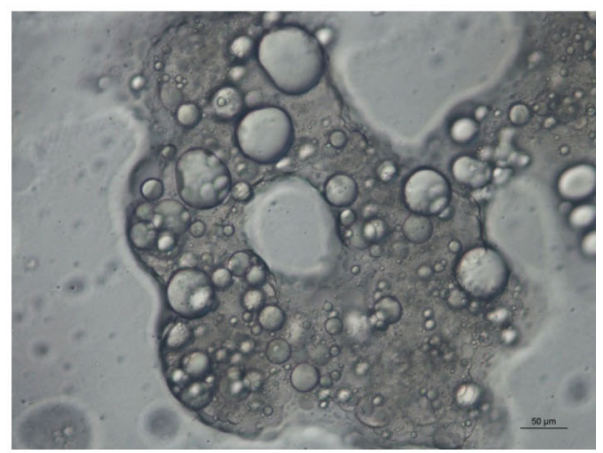

OC4. Magnificación 20X

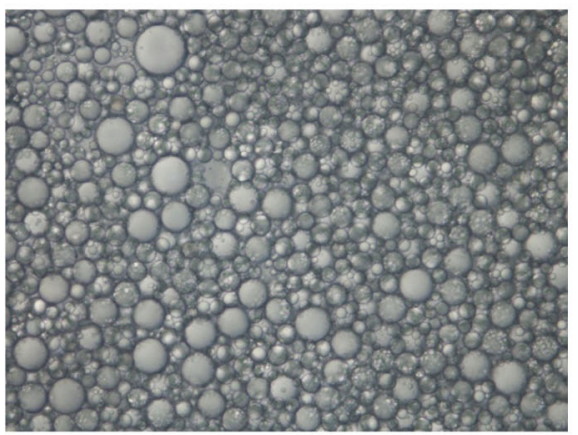

OC5. Magnificación 20X

Figura S3. Fotos del microscopio de las diferentes emulsiones para la fase oleosa de aceite de canola/octano 

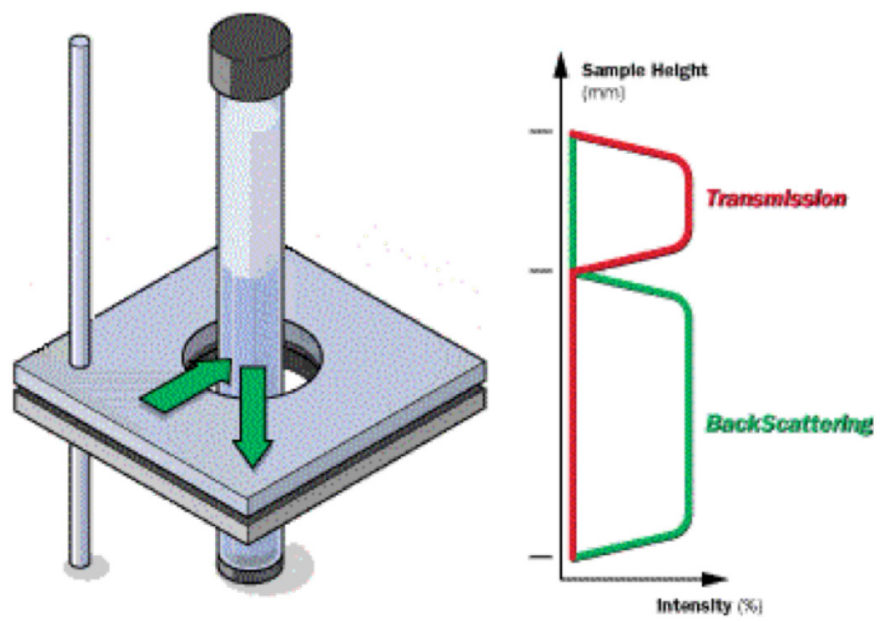

Figura S4. Principio de Funcionamiento del Turbiscan MA2000. Tomado de (Mengual, O et al; 1999)

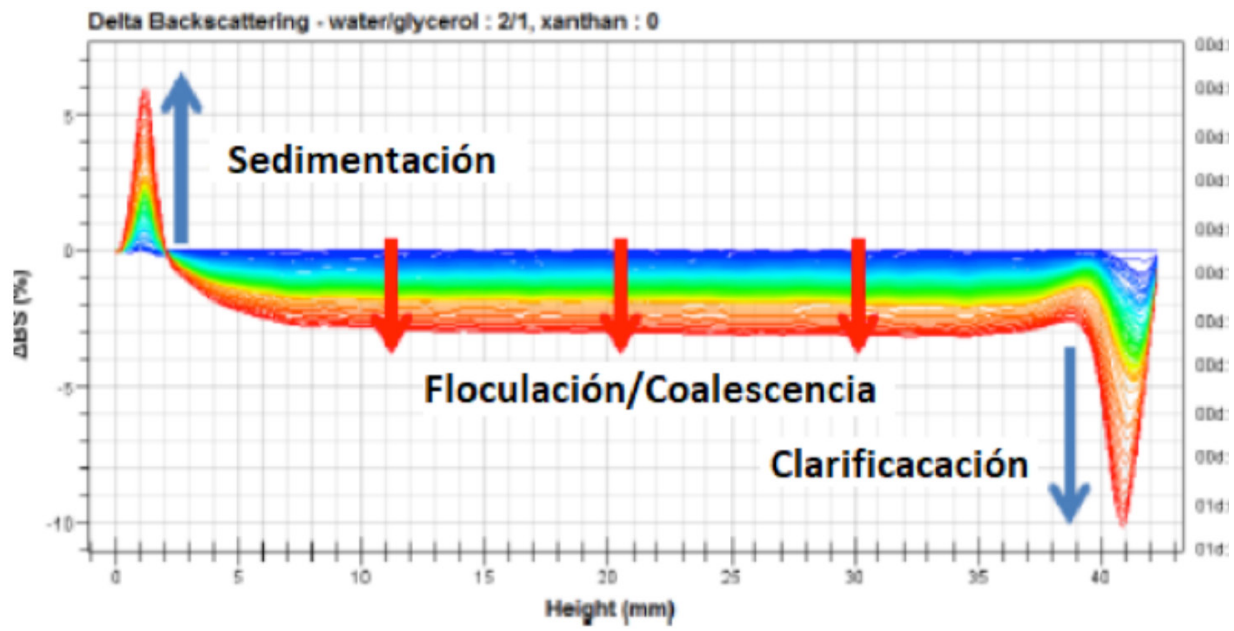

Figura S5. Reflectancia residual difusa de una emulsión de agua en aceite de una crema cosmética, con una duración de 11,5h. Tomado de Mengual, $O$ et al; (1999) 


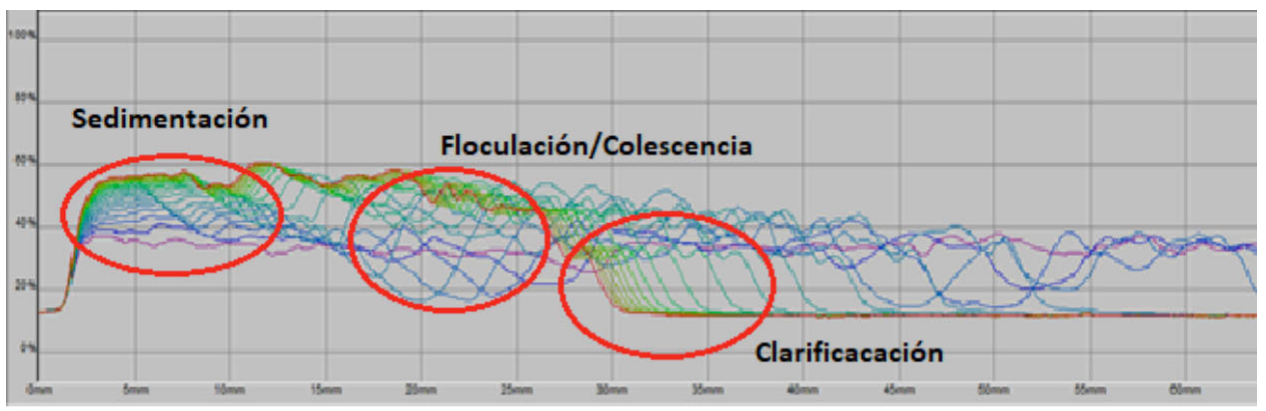

A) Formulación OC3 mezcla aceite de canola/octano

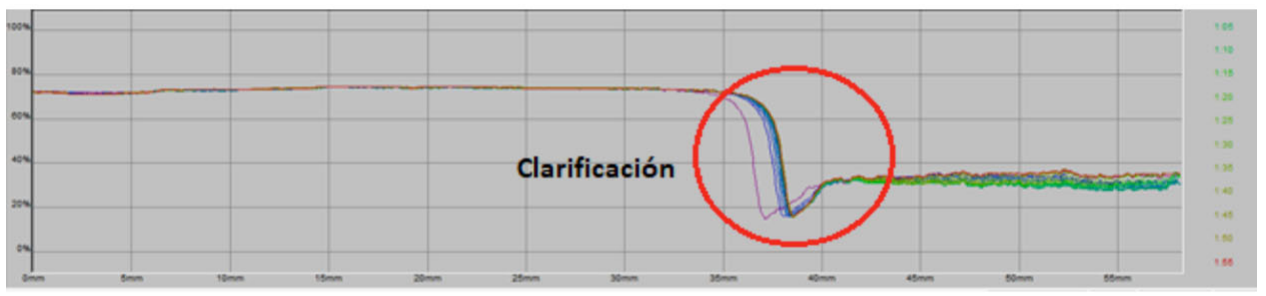

B) Formulación C1 mezcla aceite de canola

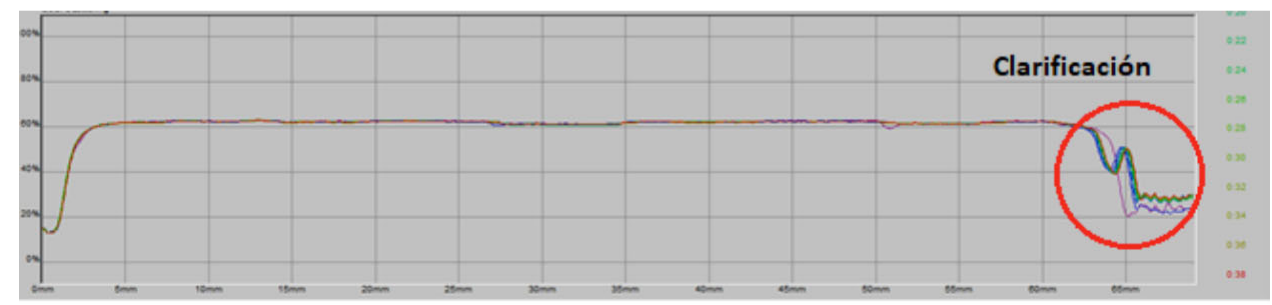

C) Formulación CP5 mezcla aceite de canola/parafina

Figura S6. Reflectancia residual difusa de diferentes emulsiones, con una duración de $2 \mathrm{~h}$. 